\title{
OBSERVING CLOUDS IN 4D WITH MULTIVIEW STEREOPHOTOGRAMMETRY
}

\author{
DAVID M. ROMPS AND RUŞEN ÖKTEM
}

Six cameras along a 12-km-diameter circle generate a 4D view of clouds at the Southern

Great Plains atmospheric observatory in Oklahoma.

S hallow cumulus clouds play a large role in Earth's current radiation balance (e.g., Hartmann 2015), and their response to global warming makes a large and uncertain contribution to Earth's climate sensitivity (Bony and Dufresne 2005). To develop accurate theories and parameterizations of shallow cloud cover, we need observations of cloud populations and their life cycles. In particular, we need measurements of the horizontal dimensions of the clouds, their elevations, their depths, the rate at which they are created, the rate at which they dissipate, and how all of these factors vary with changes to

AFFILIATIONS: ROMPS AND ÖKTEM-Department of Earth and Planetary Science, University of California, Berkeley, and Climate and Ecosystem Sciences Division, Lawrence Berkeley National Laboratory, Berkeley, California CORRESPONDING AUTHOR: David M. Romps, romps@berkeley.edu

The abstract for this article can be found in this issue, following the table of contents.

DOI:10.1175/BAMS-D-18-0029.I

In final form 22 June 2018

(C)2018 American Meteorological Society

For information regarding reuse of this content and general copyright information, consult the AMS Copyright Policy. the large-scale environment. Only observations that are high resolution relative to individual clouds in all four dimensions-space and time-can provide these needed data.

In recent decades, scanning cloud radars have been deployed in the hopes of obtaining these highresolution $4 \mathrm{D}$ observations (e.g., Kollias et al. 2007). Like all technologies, however, scanning cloud radars have their drawbacks: their scanning strategies sample with spatial and temporal resolutions that are coarse compared to the sizes and lifetimes of shallow cumuli, they have a limited range due to clear-sky attenuation, and the detectors have a limited sensitivity to reflections from small, continental cloud drops. Unfortunately, these limitations make it challenging to observe even a single cloud's life cycle with a scanning cloud radar, let alone the evolution of a population of clouds. For more detail, see the sidebar outlining other approaches to the $4 \mathrm{D}$ mapping of clouds.

In contrast, modern digital cameras can detect clouds with a high spatial and temporal resolution, with a range that is limited only by the clear-sky visibility and obscuration by foreground clouds, and with an extremely high sensitivity to cloud boundaries due to their operation in the visible spectrum. Today's 
OTHER APPROACHES TO THE 4D MAPPING OF CLOUDS

$R_{a}^{e}$ elative to stereo cameras, scanning radars have the Madvantage of being able to probe inside of clouds. Scanning precipitation radars have been used to study the life cycles of large, precipitating clouds (e.g., Minor et al. 20II; Stein et al. 2015), but their use of S- and C-band wavelengths renders even these clouds invisible to the radar in the clouds' early stages. Therefore, scanning cloud radars, which use shorter wavelengths capable of detecting cloud drops, are the preferred technology for studying shallow cumuli. Unfortunately, even a cloud radar has difficulty observing the evolution of individual cumulus clouds. A typical cloud-radar scanning strategy leaves large spaces unsampled in between slices, making it impossible to follow an individual cumulus through its life cycle. For example, the horizon-to-horizon $\mathrm{RHI}$ scan used by the scanning ARM cloud radars (SACRs; Kollias et al. 2014) samples slices that are spaced apart by $30^{\circ}$. At a range of $\sim 3 \mathrm{~km}$, which would enclose the domain covered by COGS, the spacing between these slices is $\sim 1.5 \mathrm{~km}$, which is much too large to repeatedly sample shallow cumuli, whose sizes range from hundreds of meters to $\sim 1 \mathrm{~km}$. In addition, the $\sim 5$ min required to complete a sequence of horizonto-horizon $\mathrm{RHI}$ scans is the same order of magnitude as both the lifetime of many cumuli and the advective residence times of clouds in the domain. Therefore, both the spatial and temporal resolutions of this common scanning strategy are too coarse to study the life cycle of individual cumuli. What about more focused sector scans? In the boundary layer RHI scan used by the SACRs, the scanning radars sample a sector with an $80^{\circ}$ azimuth range with slices that are separated by $2^{\circ}$. The whole $80^{\circ}$ sector is sampled once every $5 \mathrm{~min}$. To cover a domain comparable to that of COGS, we would need to consider a range of $\sim 6 \mathrm{~km}$. At a maximum range of $6 \mathrm{~km}$, the $2^{\circ}$ spacing between slices corresponds to a spacing of $\sim 200 \mathrm{~m}$. This is marginally adequate for studying shallow cumuli. The more difficult constraint is the 5 -min sampling time, which is comparable to the lifetime of a cumulus thermal and its residence time in the domain. Another challenge with cloud radars is their low sensitivity to shallow continental clouds. There are times when the cameras at the SGP site observe a parade of shallow cumuli passing overhead the CF that go undetected by the vertically pointing or scanning cloud radars. The combination of shallow and continental is particularly challenging for cloud radars: "shallow" guarantees a small liquid-water content and "continental" guarantees a high cloud condensation nuclei $(\mathrm{CCN})$ concentration and, therefore, that the small amount of liquid water is distributed among a high number of small drops. Since reflectivity is proportional to the drop radius to the sixth power, this can often make shallow continental clouds nearly transparent to the cloud radars. consumer cameras provide a typical angular resolution of $<0.1^{\circ}$ (i.e., a spatial resolution better than $50 \mathrm{~m}$ for objects as far away as $30 \mathrm{~km}$ ) and an essentially unlimited temporal resolution (frame rates can be as high as tens of hertz, although rates of about $0.1 \mathrm{~Hz}$ are used in practice). Over the oceans and in other areas with high visibility, clouds can be measured at distances of many tens of kilometers. Shallow continental clouds, even those composed of very small drops, are as easily detected by cameras as they are by the human eye. And, thanks to the vast consumer market, digital cameras are inexpensive. The high performance and low cost of digital cameras virtually guarantee that photogrammetry (measurement using photographs) will be a staple of atmospheric observation in the decades to come.

A QUICK PRIMER ON STEREO. A single digital camera can produce a beautiful time-lapse movie, but it is unable to make quantitative statements about the clouds it sees. How far away is that cloud? What is the altitude of that cloud? How big is that cloud? How fast is that cloud moving? None of these questions can be answered with images from a single camera. But, two digital cameras can answer all of these questions. This bit of magic is simple but powerful: by measuring the position of an object's image in the photographs of two widely spaced cameras, the precise location $(x, y$, and $z$ ) of that object can be calculated by triangulation. This procedure is called stereo reconstruction, and it is something our brain does with the data from our two eyes to judge the distances, sizes, and speeds of the objects around us.

To see how this works, hold a finger out in front of your face and close one eye at a time, alternating between left and right. The finger appears to move relative to the background as you switch eyes because the image of the finger is in different places on the two retinas; see Fig. 1. Mathematically, the distance to an object can be calculated by drawing a triangle: the triangle's base connects the center of the two lenses, and each of the triangle's sides connects the center of each lens to the image of the object in that lens' image plane (the retina in a human eye or the sensor array in a camera). Extrapolated out from the cameras or eyes, the two sides of the triangle will meet at the physical location of the object, so that location can be calculated using trigonometry. The measurement of distances, sizes, and speeds by way of a pair of cameras is called stereophotogrammetry, although we will use the word "stereo" as a shorthand.

The use of stereophotogrammetry for the measurement of clouds dates back more than 100 years, with 
early cloud studies making use of cumbersome theodolites to calibrate the cameras' orientations (Koppe 1896). Analog photographs were used in the stereophotogrammetry of clouds from at least the 1950s to obtain cloud positions and velocities (Malkus and Ronne 1954; Kassander and Sims 1957; Orville and Kassander 1961; Bradbury and Fujita 1968; Warner et al. 1973; Wilson et al. 1992). More recently, digital photographs have been used to calculate cloudbase heights from a pair of whole-sky imagers (Allmen and Kegelmeyer 1996; Seiz et al. 2002; Kassianov et al. 2005; Beekmans et al. 2016; Savoy et al. 2017) and to calculate the heights of cumuliform cloud tops (Zehnder et al. 2007; Damiani et al. 2008).

As mentioned above, stereophotogrammetry has the advantages of high spatial and temporal resolution, a long range, and high sensitivity. There are, of course, limitations to what stereo cameras can do. First, they cannot see inside of clouds; they can only map the surfaces visible to both cameras. Second, on days with low visibility due to haze or dense cloud cover, the detection range of cameras can be greatly limited. Third, the algorithms for reconstructing clouds are only able to operate when there are feature points-unique identifiable features-for the algorithms to grab a hold of, and so smooth stratiform clouds cannot be measured by stereophotogrammetry. And, fourth, since the cameras currently deployed work passively in the visible, they are able to measure clouds only in the daytime.

Despite these limitations, stereophotogrammetry can provide observations that no other instrument can. And, they are a powerful complement to other traditional instruments. For example, the new stereo cameras that we report on below can track shallow clouds for several kilometers before and after they arrive over zenith-staring radars, lidars, and radiometers. For typical wind speeds of $5-10 \mathrm{~m} \mathrm{~s}^{-1}$, this corresponds to $10-20 \mathrm{~min}$ of cloud tracking by the stereo cameras. This can provide life cycle context for other measurements, enabling classification of radar, lidar, and radiometer data into observations during the developing, mature, or dissipating phases of convective clouds.

\section{HISTORY OF BERKELEY'S STEREO PROJ-}

ECT. The cloud stereo project at the University of California, Berkeley (UC Berkeley), and the Lawrence Berkeley National Laboratory can be traced back to the first author's estimation of cloud-top vertical velocities while vacationing on the Yucatan Peninsula in the summer of 2008. Holding a thumb out at arm's length in the direction of an isolated cumulonimbus, the depth of the subcloud layer was first measured in thumb widths. Since the height of the cloud base is equal to the lifting condensation level (LCL), and since the LCL is a function of relative humidity (Romps 2017), a thumb width can be converted to meters using the relative humidity from the local weather report. Lifting the thumb to cloud top, holding the arm still, and counting off seconds, it was then possible to estimate cloud-top vertical velocities. This 


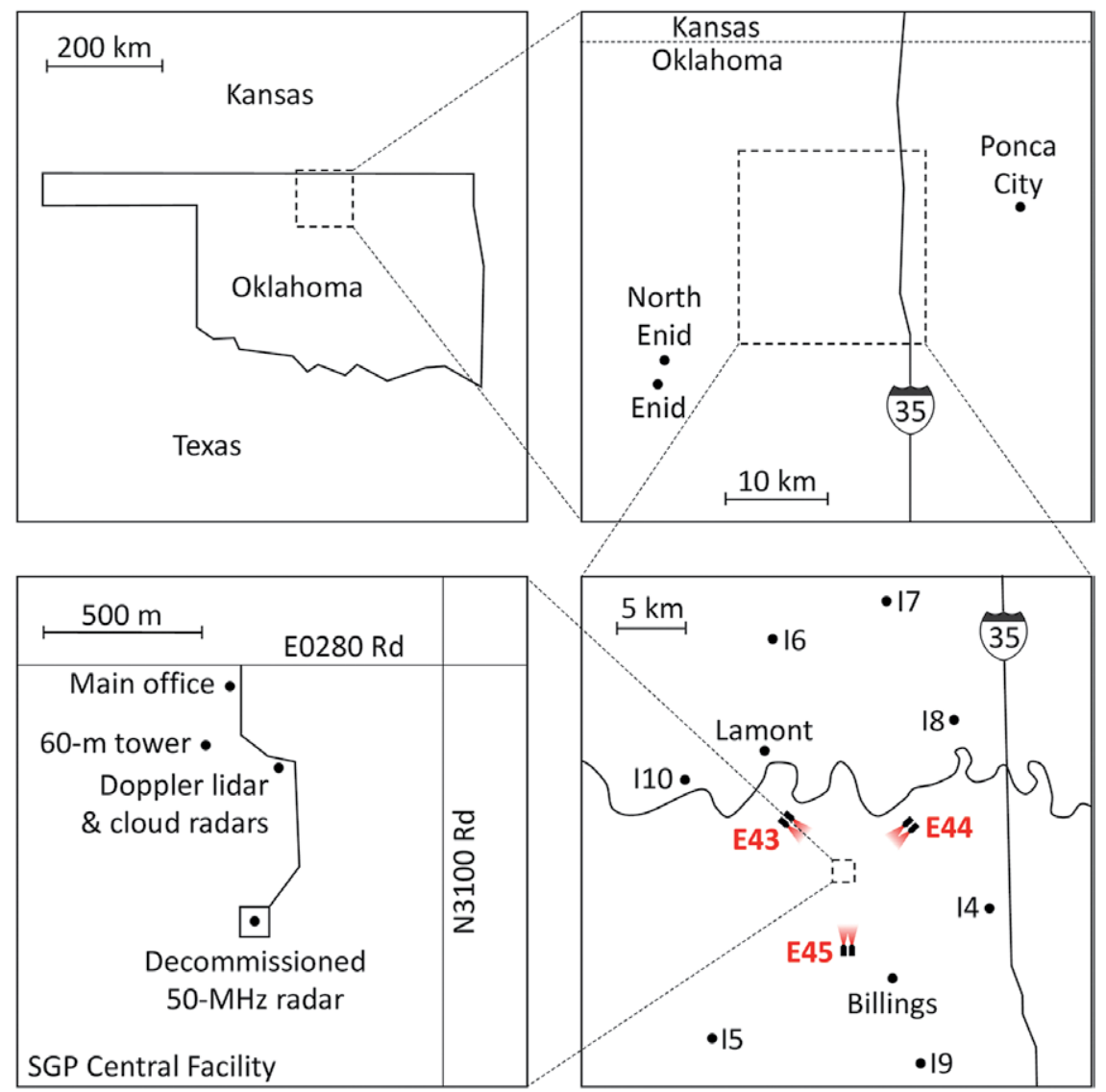

FIG. 2. Map of the stereo-camera pairs at sites E43, E44, and E45 ringing the SGP CF.

was not a research-grade measurement, confounded as it was by the horizontal motion of the clouds, but it gave the right ballpark: $\sim 10 \mathrm{~m} \mathrm{~s}^{-1}$ of cloud-top ascent for fast-moving updrafts. Most importantly, it illustrated the power of photogrammetry, planting the seed of future research.

In the spring of 2011, Dale Durran visited UC Berkeley and raised the question of whether largeeddy simulations (LESs) were correctly simulating the speeds with which cumulonimbi rise through the upper troposphere. The discussion quickly shifted to how one could validate these upper-tropospheric ascent speeds when the existing measurements are so sparse. Aircraft, even research aircraft, tend to avoid strong updrafts in the upper troposphere. Vertically pointing radar can gather useful data, but it is a challenge to get the appropriate combination of resolution and range, coupled with reliable algorithms to subtract off the free-fall speed of the reflecting hydrometeors. And radar is expensive. But, what if photogrammetry (i.e., a digital version of the analog thumb measurements) could measure these speeds on the cheap? Better yet, what about stereophotogrammetry, which can measure the positions and speeds of objects directly through stereo reconstruction?

To pursue stereophotogrammetry, we began looking for a place to install a pair of cameras. As luck would have it, Paquita Zuidema had just installed a camera on the roof of the Rosenstiel School of Marine and Atmospheric Science (RSMAS) at the University of Miami as part of her Cloud-Aerosol-Rain Observatory (CAROb). That camera looked out over Biscayne Bay, providing an unobscured view of crisp, white cumuliform clouds set against the background of a clear, blue sky. To enable stereophotogrammetry, we installed a second camera about $1 \mathrm{~km}$ away on the roof of the Maritime and Science Technology (MAST) Academy, a public high school in Miami, Florida, in the spring of 2012. By comparison to CAROb lidar, the stereo reconstructions of shallow clouds from the paired RSMAS and MAST cameras were deemed accurate to within a few tens of meters (Öktem et al. 2014).

Having validated the stereo setup, we turned to the original question: do LESs correctly capture vertical velocities in the upper troposphere? To address this, we used the RSMAS and MAST cameras to measure the sizes, heights, and vertical velocities of 32 cloud thermals between April 2013 and July 2014. Those data showed that the dominant balance in the vertical momentum equation for deep cloud updrafts is between buoyancy and drag (Romps and Öktem 2015), and not between buoyancy and acceleration as had been proposed by Sherwood et al. (2013). Since their acceleration is so much smaller than their buoyancy, cloud thermals can be described colloquially as "being sticky" or "rising viscously." These results-both the magnitude of the vertical velocities and the dominant balance in the momentum equation-have proved consistent with 
large-eddy simulations (Romps and Charn 2015; Hernandez-Deckers and Sherwood 2016; Morrison and Peters 2018).

In the next phase of the UC Berkeley stereo project, we shifted our attention to the continental interior with the Measuring Clouds at SGP with Stereo Photogrammetry (MCSP) campaign at the Department of Energy (DOE) Atmospheric Radiation Measurement (ARM) Program's Southern Great Plains (SGP) Central Facility (CF). As shown in Fig. 2, the SGP CF is located in north-central Oklahoma in between the towns of Lamont and Billings. The MSCP campaign began in April of 2014 with the siting of two westward-facing cameras at the CF: the northern camera was affixed to the $60-\mathrm{m}$ tower and the southern camera was erected on a portable tower next to the site of the decommissioned 50 $\mathrm{MHz}$ radar wind profiler. This ongoing campaign reconstructs points on the eastern sides of clouds in a triangular domain to the west of the CF. The product from these cameras is the Point Cloud of Cloud Points (PCCP) product, which is a collection of the 3D positions of cloud feature points, available on the ARM data archive (www.arm.gov/data). Using these data, the widths and elevations of clouds can be calculated. Although not very user friendly, this type of PCCP product serves as the foundation for all other stereo products, including the gridded cloud product described in the next section.

COGS. In July of 2017, six new cameras were installed at remote locations ringing the Central Facility to develop the Clouds Optically Gridded by Stereo (COGS) product (logo shown in Fig. 3). COGS is a gridded dataset that identifies the patches of atmosphere that are inside clouds. To generate such a dataset, we need stereo reconstructions of the clouds from all sides. To this end, the six cameras are grouped into pairs at a distance of $\sim 6 \mathrm{~km}$ from the $\mathrm{CF}$ and with relative azimuths of $\sim 120^{\circ}$ between

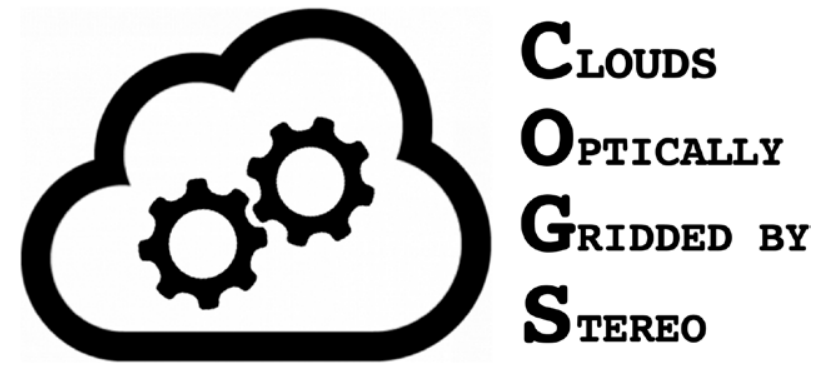

Fig. 3. The COGS logo.

pairs. The bottom-right panel of Fig. 2 shows the locations of the camera pairs, which have been labeled sites E43, E44, and E45. Despite the nomenclature, each of these "sites" consists of two separate locations separated by $500 \mathrm{~m}$, with each location hosting a stand-alone camera. Because these six cameras are at remote locations, they are powered by solar photovoltaics and they communicate with ARM servers using a cellular transceiver. Figure 4 shows one of these setups. The "From camera to COGS: How it works" sidebar gives a more detailed description of the components.

Each camera uses a wide-angle lens that provides a $\sim 70^{\circ}$ field of view, and each pair of cameras reconstructs feature points in a roughly pyramidal volume

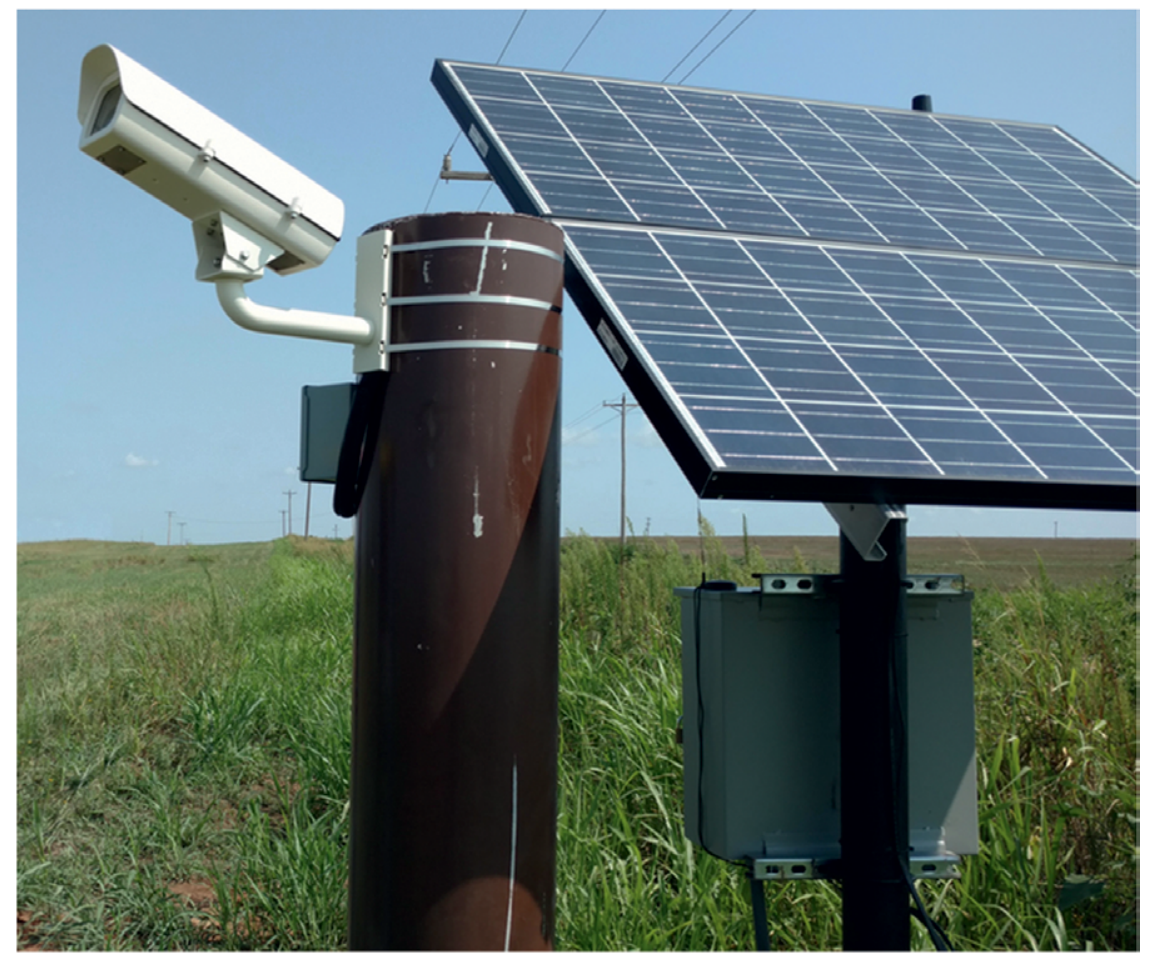

FIG. 4. One of the six cameras that compose the stereo ring. The pole-mounted camera, tilted approximately $18^{\circ}$ from the ground, is powered by two solar photovoltaic panels connected to a lead-acid battery and communicates with the ARM servers using a cellular network. 
Each of the six cameras is powered Ey a deep-cycle battery charged by two 145-W solar panels. An electrical enclosure holds a charge controller (which regulates power between the solar panels, the battery, the camera, and other electrical loads), a cellular modem (which provides 24-7 network connectivity), a computer (which instructs the camera to capture images at 20-s intervals and temporarily stores images and auxiliary data), and a global positioning system (GPS) receiver (to ensure that the computer's clock is accurate). The camera enclosure contains the camera (a 5-megapixel security camera), a fan (to cool the camera during hot summer days), and thermometers (to monitor the camera temperature and to trigger the fan as needed).

Once the cameras have been installed, there are several steps that must be followed to generate a gridded cloud product. First, the cameras are subjected to an internal calibration, by which the camera's optical parameters are determined. These optical parameters include the focal length, any lateral offset of the imaging array, and the barrel distortion of the optics. These parameters can be determined by collecting images of a planar grid held at different angles and positions relative to the camera; in practice, we use a checkerboard from a game shop. The next step is the external calibration, whereby each camera's position (latitude, longitude, and altitude) and orientation (pitch, roll, and yaw) are determined. The position is needed to an accuracy of a few meters, and the three Euler angles are needed to an accuracy of hundredths of a degree. The position is determined through a combination of in-the-field GPS measurements and Google Earth. The Euler angles are measured by the measurement of stars, planets, and/or known landmarks in the camera's field of view (Öktem et al. 2014; Öktem and Romps 2015). The cameras are mounted onto a sturdy pole cemented into the ground to ensure that these angles do not change after they are measured.

Images are captured from all six cameras every $20 \mathrm{~s}$ with a time synchronization that is accurate to much better than I s. This generates six synchronized time-lapse videos. The six cameras are grouped into three pairs, with the baseline between cameras in a pair equal to $500 \mathrm{~m}$, and with the three pairs located $6 \mathrm{~km}$ from the CF at roughly $120^{\circ}$ angles from each other. Within each pair, cameras are labeled $A$ and $B$. For each image captured by $A$, an edge-detection algorithm identifies the locations in the image of hundreds of distinctive cloud features. An algorithm is then used to match the location of each feature point in image $A$ with its corresponding location in image B (Öktem and Romps 2015). This is accomplished through block matching: the algorithm searches along the epipolar line in image $B$ for a small, square subset of image $B$ that has a high spatial correlation with the square subset centered on the feature in image A. In practice, hierarchical block matching is used to increase both accuracy and computational efficiency: the approximate location of the feature point is found in image $B$ using coarsened images, and then the location is refined by progressively reducing the applied coarsening. Once the feature points are matched, stereo reconstruction (basically, triangulation) is used to calculated the 3D position (i.e., latitude, longitude, and altitude) of each feature point. For each pair of cameras, this produces the PCCP product at 20 -s intervals. For each time interval, the points are joined together into surfaces, and the surfaces from the three pairs of cameras are stitched together to make a closed surface. Discretizing space into cubes that are 50-m wide, grid points that are inside these closed surfaces are labeled cloudy. This produces the COGS product. whose vertex lies about $300-400 \mathrm{~m}$ in front of the two cameras. Since the entire volume of a cloud can be mapped only if it is viewed from all sides, we can only generate COGS in the volume of overlap among those three pyramids. This volume of overlap is illustrated in Fig. 5, which maps the ceiling on the overlap volume. Since this system is designed for shallow clouds, the maximum potential height of reconstruction is $6 \mathrm{~km}$, with the vast majority of the domain able to reconstruct points exceeding $2 \mathrm{~km}$ in altitude. The data are gridded within a cubic domain that is $6 \mathrm{~km}$ on a side, centered on the location of the Doppler lidar $\left(36^{\circ} 63^{\prime} 19^{\prime \prime} \mathrm{N}, 97^{\circ} 29^{\prime} 11^{\prime \prime} \mathrm{W}\right)$, and with the bottom of the domain at ground level. Grid points that are outside the reconstructed volume are tagged with missing values.

Within this $6 \mathrm{~km} \times 6 \mathrm{~km} \times 6 \mathrm{~km}$ domain, the reconstructed feature points from the three pairs of cameras are connected into surfaces, and then the three sets of surfaces are stitched together to generate $3 \mathrm{D}$ volumes. This is all done algorithmically with minimal human intervention; see the "From camera to COGS" sidebar for more detail. The cubic domain is then sliced into an isotropic 50 -m grid; grid points inside a cloudy volume are labeled 1 and all other grid points (within the reconstructable volume) are labeled 0 . This is repeated for every sextuple of images, which are generated every $20 \mathrm{~s}$. The result is the $4 \mathrm{D}$ gridded cloud COGS product $(50 \mathrm{~m} \times 50 \mathrm{~m} \times 50 \mathrm{~m} \times 20 \mathrm{~s})$. A rendering of a sample COGS snapshot during a shallow-cumulus case is shown in Fig. 6.

Although the six cameras were installed in July 2017, it took several weeks to identify and replace faulty hardware and to fully calibrate the cameras. The last issue was resolved at 1800 UTC 31 August 2017, which is the time that the stereo ring can be considered to have been "turned on." As luck would have it, the stereo ring turned on in the middle of 
a shallow cumulus case. Figure 7 displays the cloud cover (nonwhite areas) and cloud thickness (see the color bar) of clouds from the stereo cameras' first reconstruction of clouds at 1800 UTC 31 August 2017. With the COGS data, this calculation of cloud thickness is trivial: it is simply the thickness of the cloudy volume at each point in the horizontal domain. The projected cloud fractional area is about $15 \%$ at this time, with a maximum thickness of about $300 \mathrm{~m}$. Based on the aspect ratios evident in Fig. 7-wider than they are thick-these are cumulus humilis. Some COGS data files are available for download from the ARM archive (https://iop.archive.arm.gov/arm -iop/0eval-data/romps). The browser will be directed to the download page after signing in at the prompt.

\section{VALIDATION AGAINST}

LIDARS. From the time the cameras were activated on 31 August, there were 6 days with shallow cumulus through the remainder of the 2017 calendar year: $31 \mathrm{Au}$ gust, 3 and 10 September, 2 and 10 October, and 18

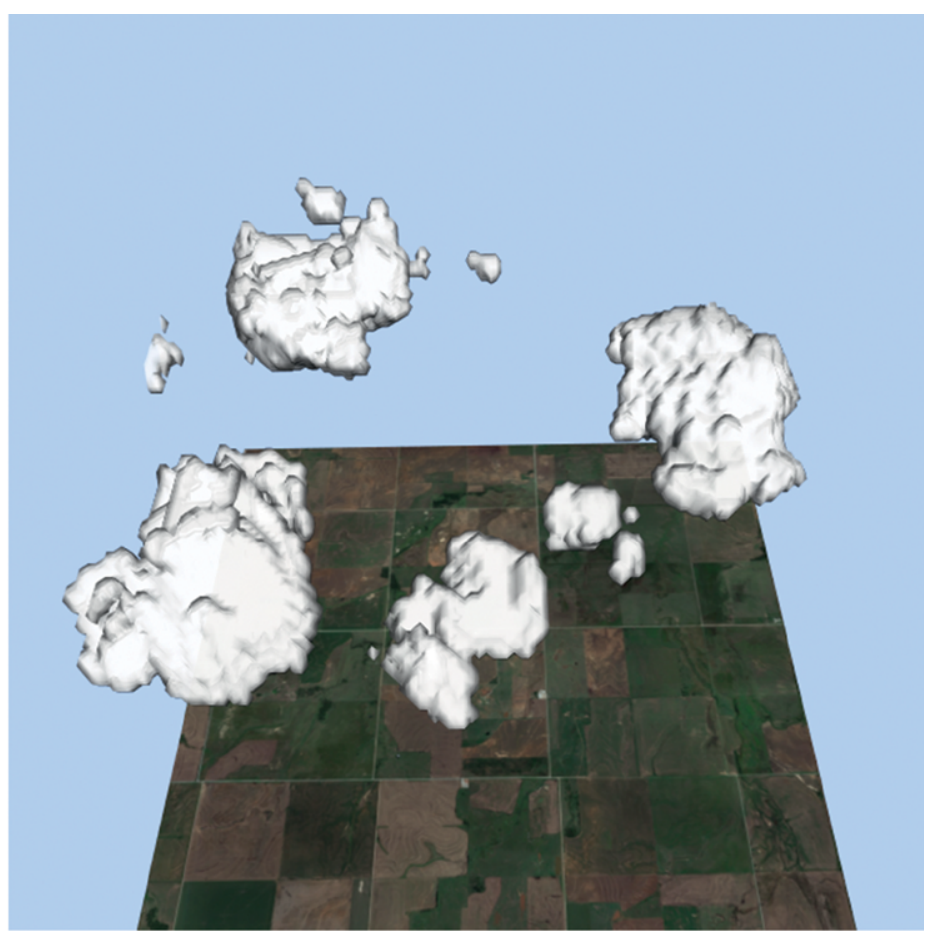

Fig. 6. Snapshot of the COGS 4D reconstruction of clouds over a 6-km-wide square domain centered on the SGP CF.
Maximum altitude observed by all six cameras

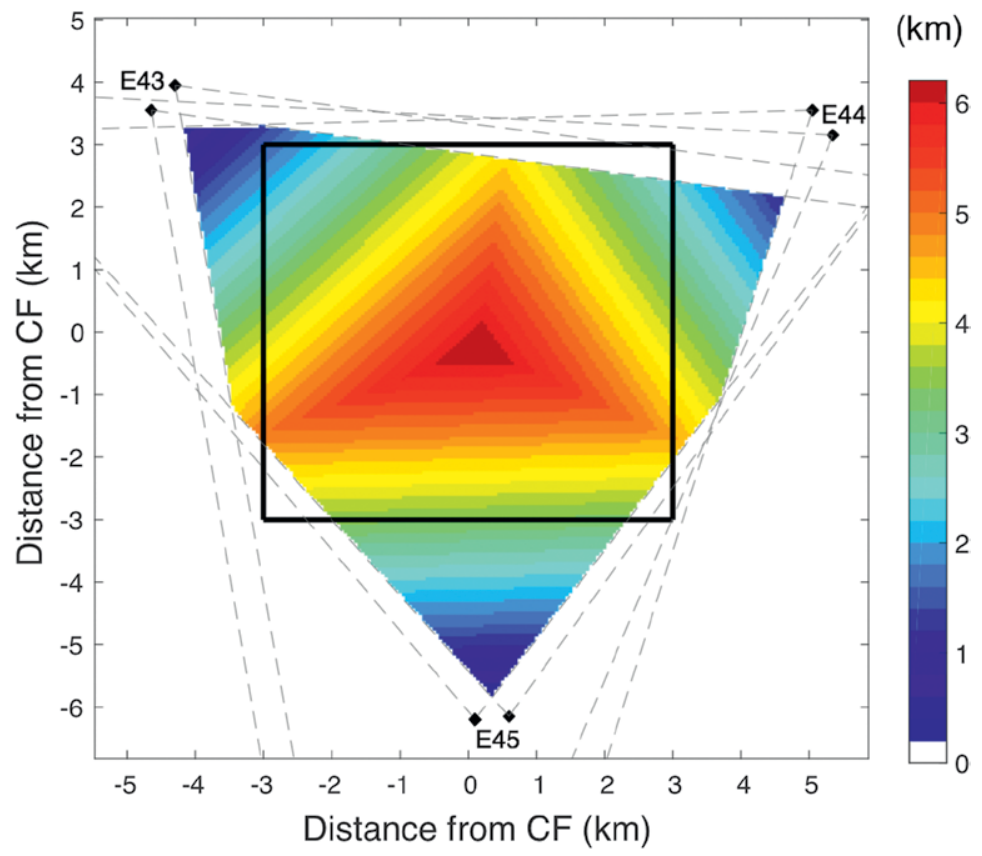

FIG. 5. Maximum altitude that is visible to all six cameras. The black square denotes the current COGS domain (i.e., the region for which the 4D gridded product is generated). The dashed gray lines mark the edges of each camera's field of view.

November. The Active Remote Sensing of Clouds (ARSCL) product, which gives the heights of cloud layers from a combination of lidar and radar data, is available in the ARM data archive only up through the end of October, so we focus here on the first five of those days. Over these five days, there are hundreds of individual cumulus clouds in the COGS dataset. Figure 8 shows the time series of the projected shallow-cloud area fraction calculated from the COGS product every $20 \mathrm{~s}$. On two of the five days, the stereo data are partial: on 31 August, the stereo ring turned on at about 1800 UTC; on 10 October, a loose cable in one camera led to an outage of the stereo ring before about 1800 UTC. During those months, Oklahoma observed central daylight time (CDT), which lags UTC by 5 h. From these time series, we see that the shallow cumuli appear in the afternoon ( 1200-1600 CDT) and disappear a few hours later (1430-1800 CDT). The maximum daily projected cloud fraction ranges from as little as $\sim 10 \%-20 \%$ in the September cases to as much as $\sim 60 \%-70 \%$ in the October cases. The high-frequency variations in 
the area fraction are due primarily to clouds entering and exiting the 6-km-wide domain. For a typical horizontal wind of $5-10 \mathrm{~m} \mathrm{~s}^{-1}$, the residence time of a cloud in the domain is about $10-20 \mathrm{~min}$, which is the order of magnitude of the duration of spikes in the cloud-cover time series.

Figure 9 plots the time series of the mean cloud base from COGS on the five selected days. The mean cloud base is calculated every $20 \mathrm{~s}$ as the average of the heights of the lowest cloudy grid box in each cloudy COGS column. Consistent among these cases is the fact that the cloud base rises throughout the afternoon. This is caused by the decreasing relative humidity of the surface air, which increases the LCL.

Shown in red in Fig. 9 are the time series of the cloud-base heights from ARSCL, which provides an estimate of cloud base from the combined output of a laser ceilometer and a micropulse lidar (Clothiaux et al. 2000, 2001). For the red data points in Fig.

9, we use the cloud_base_best_estimate variable in the sgparsclkazrbnd1kolliasC1.c0.YYYYMMDD.000000. nc files on the ARM data archive.
Thickness of clouds at 18:00 UTC on August 31, 2017

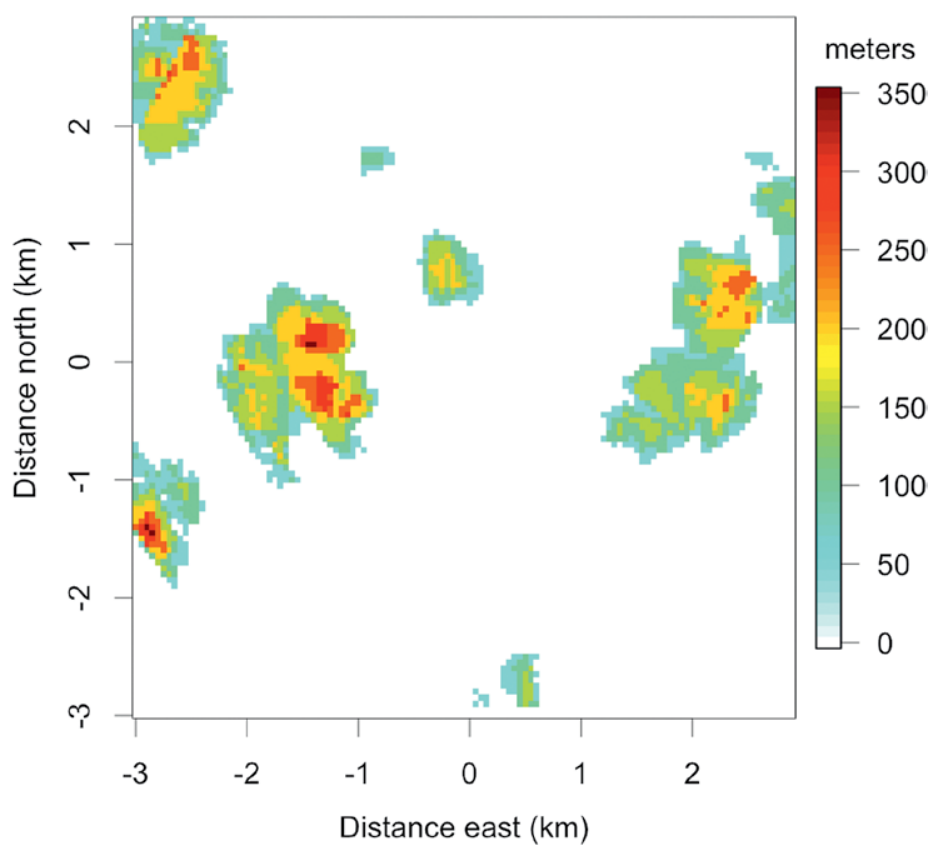

Fig. 7. Thickness of clouds calculated from COGS at the moment the stereo ring turned on at 1800 UTC 3I Aug 2017.

The ARSCL cloud-base time series is noisier than the stereo cloud-base time series. The standard deviation of the difference in minute-to-minute

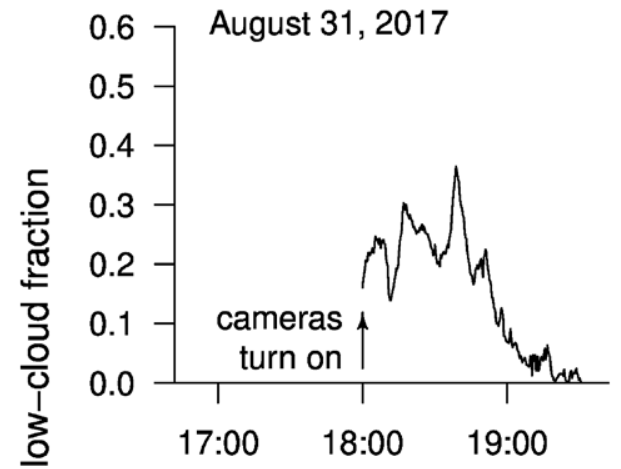

क

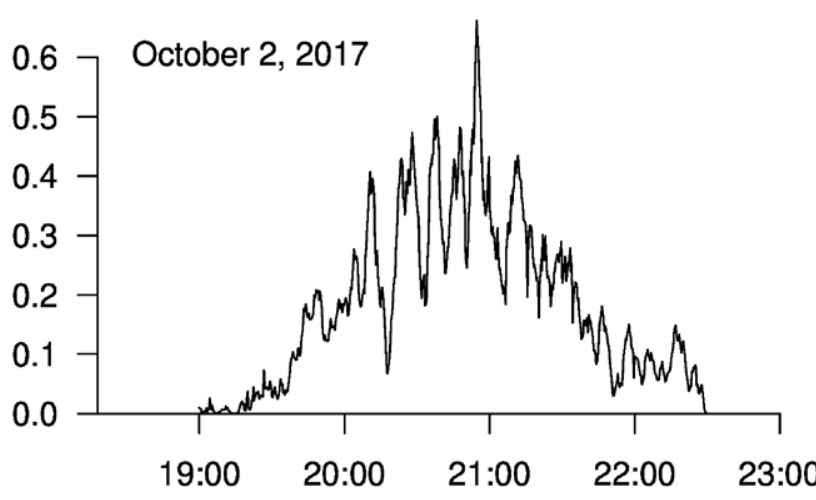

September 3, 2017

September 10, 2017
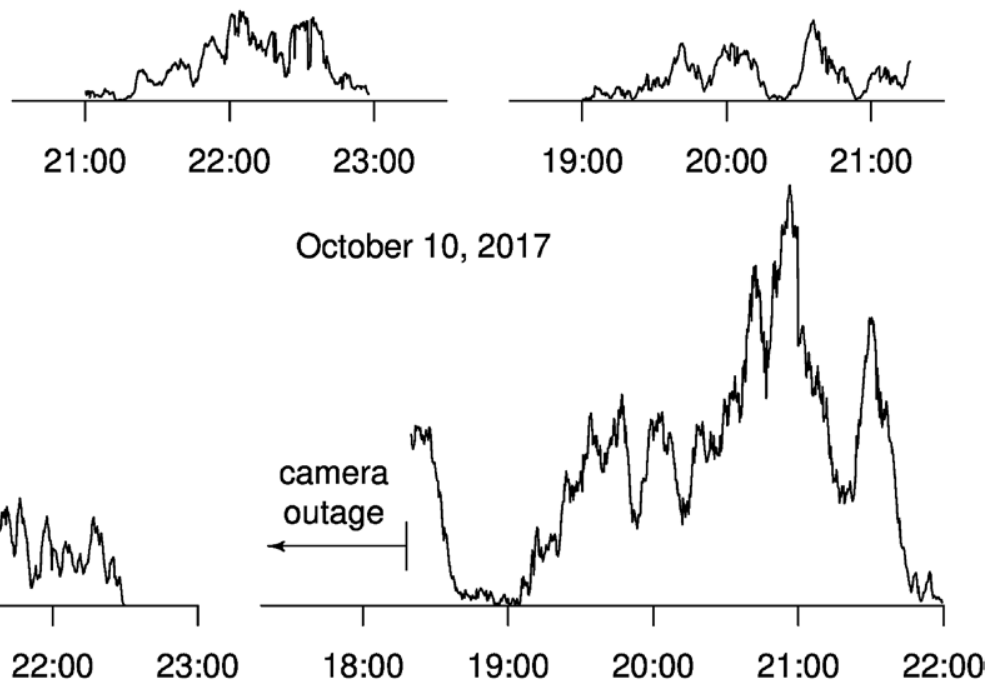

Time in UTC

FIG. 8. Time series of the COGS-projected shallow cloud fraction on the five selected days with shallow cumulus. 

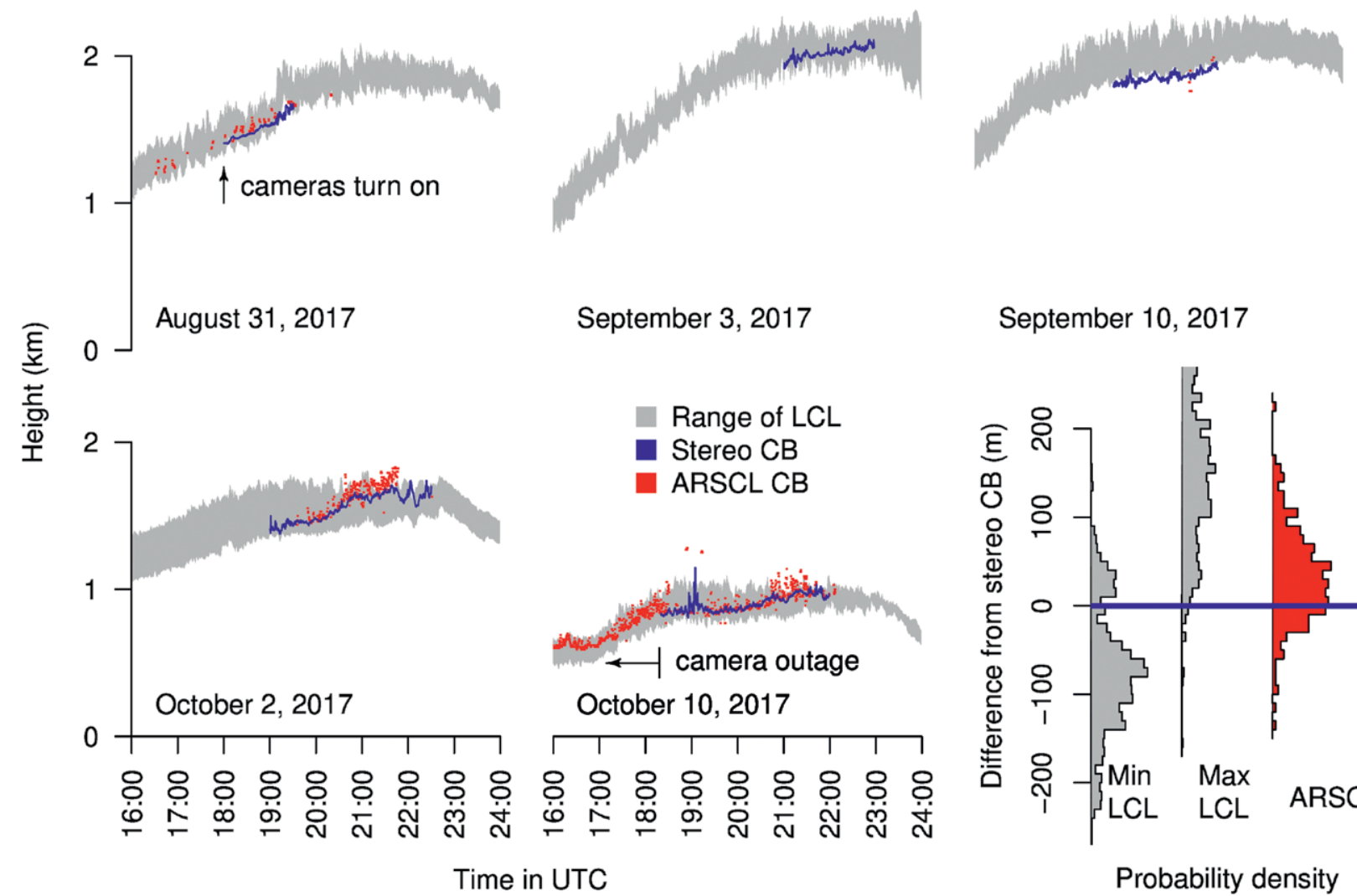

September 10, 2017

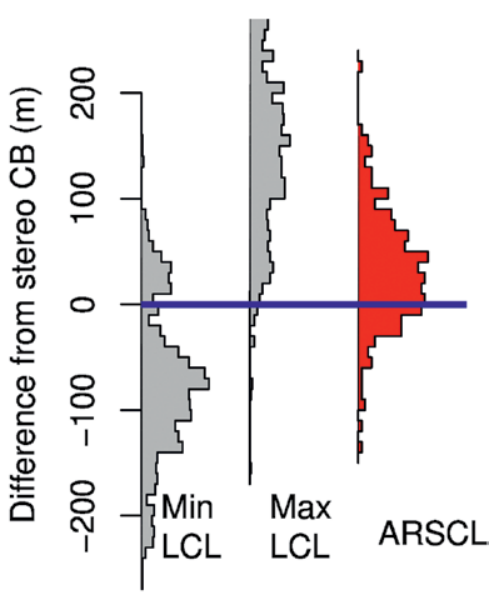

Probability density

FIG. 9. For five days with shallow cumulus, the time series of the range of LCLs calculated from the six pairs of thermometers and hygrometers (gray), the cloud base extracted from COGS (blue), and the cloud base reported by ARSCL (red). The last panel shows the PDFs of the minimum LCL, the maximum LCL, and the ARSCL cloud base relative to the contemporaneous COGS cloud base.

cloud-base heights (restricting to the data shown in Fig. 9) is $20 \mathrm{~m}$ for the stereo cameras and $47 \mathrm{~m}$ for ARSCL. That the ARSCL cloud bases have a higher variability should not come as a surprise: the ARSCL cloud-base height comes primarily from the vertically pointing ceilometer, which samples only a single piece of a cloud at a time. The stereo cameras, on the other hand, measure cloud bases throughout a $6-\mathrm{km}$-wide square domain, the average of which will be less variable.

In each of the time series plots, the gray bands denote the range of LCL values calculated from six thermodynamic sensors: the Surface Meteorology System (MET) and the Temperature, Humidity, Wind, and Pressure Sensors (THWAPSs) at 2-m elevation, the southeast and west sensors at $25 \mathrm{~m}$ on the $60-\mathrm{m}$ tower, and the southeast and west sensors at $60 \mathrm{~m}$ on the $60-\mathrm{m}$ tower. We see that the stereo cloud bases lie almost exclusively within that LCL band, as we would expect for shallow cumulus. Also, the stereo cloud base and ARSCL cloud base are nearly coincident, indicating that the stereo reconstructions are accurate. The last panel in Fig. 9 shows the probability density functions (PDFs) of the minimum of the LCLs calculated from the six sensors, the maximum of the six LCLs, and the ARSCL cloud base, all relative to the stereo cloud base. The stereo cloud base lies largely between the minimum and maximum recorded LCLs, and in the middle of the distribution of ARSCL cloud bases.

Among these five days, the Doppler lidar (DL) was in a range-height indicator (RHI) scanning mode on 31 August and 10 September. During the afternoons on those days, the Doppler lidar alternated between 27 min of RHI scanning and 3 min of zenith staring. Each DL scan took about 1 min and spanned zenith angles from $-45^{\circ}$ to $45^{\circ}$. These files are stored on the ARM data archive as sgpdlrhi2C1.b1.YYYYMMDD. HHMMSS.cdf. We identify cloudy points as locations where the intensity variable exceeds 1.2 , corresponding to a signal-to-noise ratio exceeding 0.2. Figure 10 shows an example from 31 August at a time when a cloud is drifting overhead the DL. The black dots show the DL cloud points. The gray boxes show where, in the plane of the DL scan, COGS identified the air as cloudy during the $\sim 1$-min DL scan. 
Figure 11 shows the PDF of DL cloud detections as a function of their distance from a COGS cloud edge. Of all the DL cloud detections, $50 \%$ are inside a COGS cloud, and $80 \%$ are either inside a COGS cloud or within $100 \mathrm{~m}$ of a COGS cloud edge. The existence of DL cloud detections outside a COGS cloud may be due to many factors. First, there is an uncertainty in the stereo reconstruction of a cloud feature that is around $50 \mathrm{~m}$; this is why we do not attempt to grid COGS on a grid any finer than this. Some additional error is generated by fitting a surface to the reconstructed cloud features: where the surface is interpolated between reconstructed points, the COGS cloud surface may cut inside the actual cloud. Another potential source of discrepancy is the intrinsic ambiguity as to what counts as a cloud. A lidar can be sensitive to thin wisps of clouds that may not be sufficiently optically thick to be reconstructed from the cameras' images. These thin wisps can be part of the periphery of cloud, which may account for

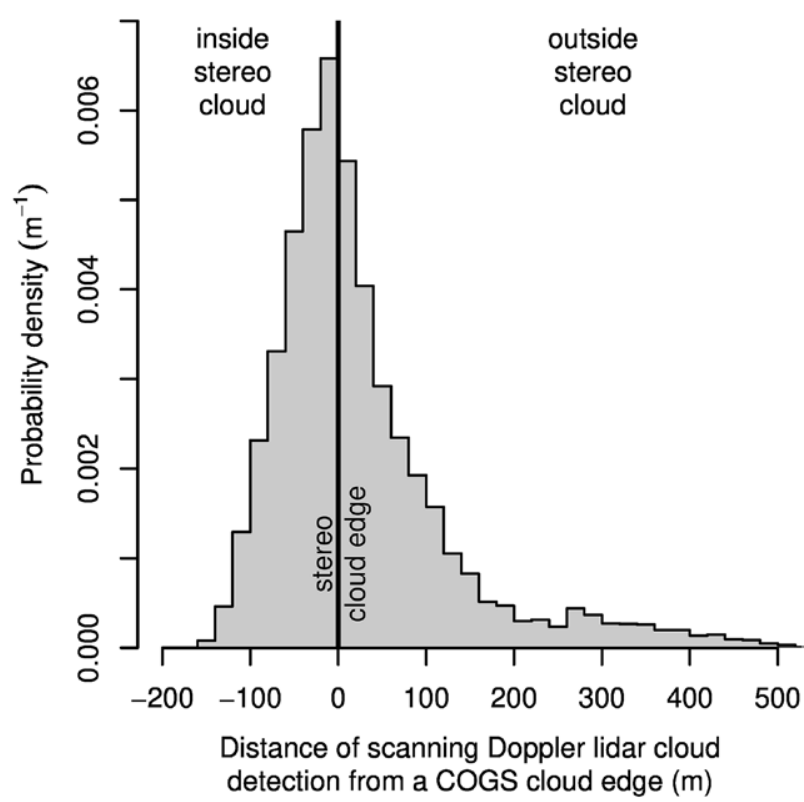

FIG. II. The PDF of scanning Doppler lidar cloud points relative to the nearest COGS cloud boundary.

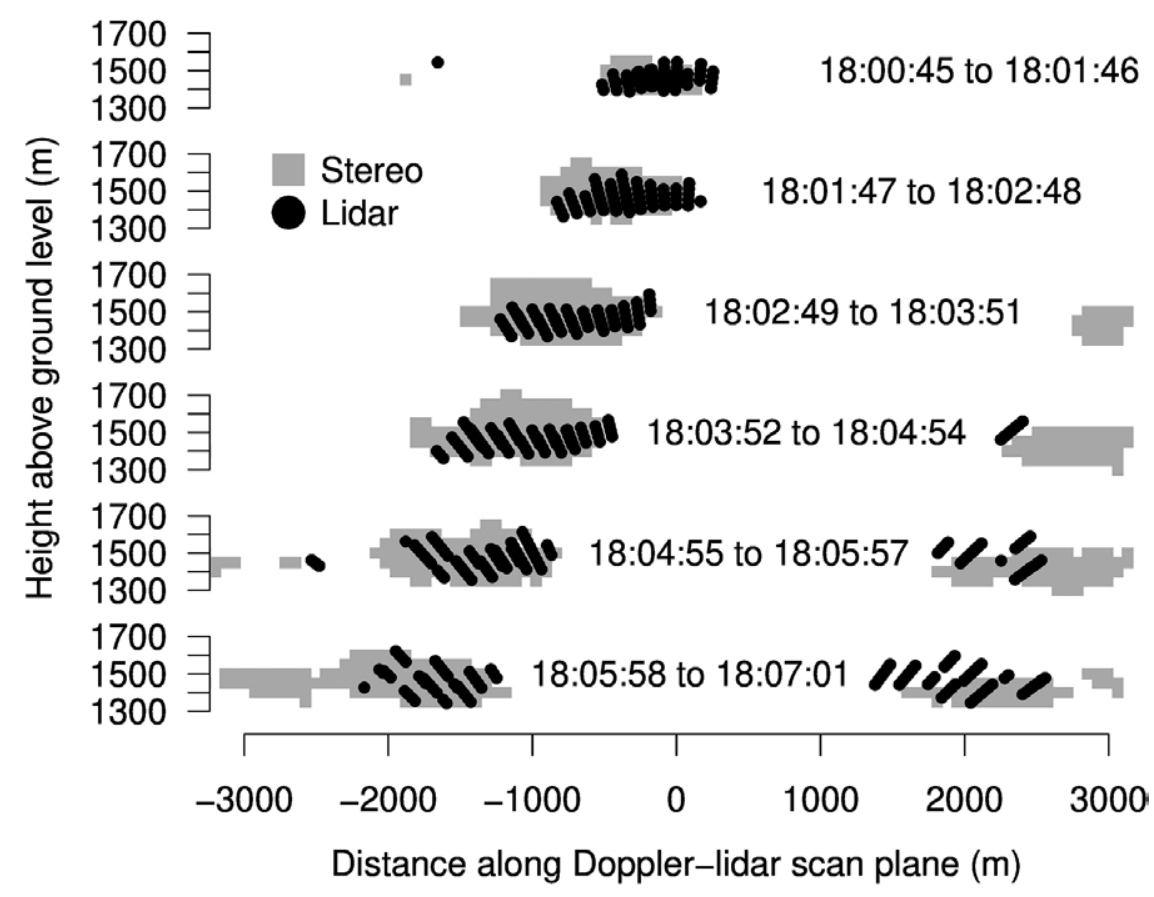

FIG. I0. A cumulus drifts over the CF on 3 I Aug 2017 and is observed by the scanning DL (black circles) and the stereo cameras' COGS product (gray squares) during each $\sim 1-$ min DL scan in the plane of the DL's scan.

the $30 \%$ of DL detections that lie within $100 \mathrm{~m}$ of a COGS cloud surface. Thin wisps can also be standalone remnants of old and decaying clouds, which may account for the $20 \%$ of DL detections that lie farther away from COGS clouds. This suggests that the COGS cloud boundaries are accurate to $\sim 100 \mathrm{~m}$, with some small, wispy clouds-representing a small minority $(20 \%)$ of the total cloud volume-left undetected.

DISCUSSION. We have described here a ring of six cameras installed on a $12-\mathrm{km}$-diameter circle at the Southern Great Plains site in Oklahoma. These cameras are constantly generating the PCCP product whenever there are identifiable cloud features in their field of view. This dataset forms the foundation of all other stereo data products. During times of shallow cumulus, the PCCP data are processed into the COGS product, which tags the atmosphere as cloudy or clear on a 50-m grid every $20 \mathrm{~s}$.

From prior experience with stereo cameras in Miami and Oklahoma, the uncertainty on the reconstruction of a feature point at low altitudes less than $10 \mathrm{~km}$ from a camera pair is about $50 \mathrm{~m}$. Some additional uncertainty is introduced by interpolating those points to surfaces and then those surfaces to volumes. Based on a comparison of the COGS data with the ARSCL and Doppler lidar, the uncertainty in the boundaries of the COGS cloud 
volumes is about $100 \mathrm{~m}$. For many purposes, including quantifying the sizes, altitudes, thicknesses, and lifetimes of shallow cumuli, this is an acceptable degree of uncertainty.

Because COGS is a high-resolution, high-frequency gridded product, there are many derivative products that can be generated from it. For example, it is straightforward to calculate from COGS the time series of cloud-base height, profiles of cloud area fraction, and the distribution of cloud sizes and thicknesses. Horizontal winds in the cloud layer are easily calculated by finding the horizontal displacement that gives the best spatial correlation from one time frame to the next. The growth and decay of individual clouds can be visualized and quantified by applying standard tracking algorithms to the COGS data. Cloud-top vertical velocities can be calculated by tracking individual clouds and recording the height of the highest contiguous cloudy pixel.

It is envisioned that COGS will be particularly useful in combination with the other instruments at the SGP site. The 12-km-wide circle along which the cameras were installed was chosen to have the SGP CF instruments at its center. For typical horizontal wind speeds, COGS sees a cumulus cloud for $\sim 5-10 \mathrm{~min}$ before and $\sim 5-10$ min after the cloud arrives overhead the CF's vertically pointing instruments. Therefore, COGS can place into context the data from the CF's vertically pointing lidars and radars: COGS can indicate where in a cloud those instruments are sampling (e.g., edge vs center) and when in a cloud's life cycle those instruments are sampling (e.g., developing, mature, or dissipating). These data are likely to make valuable contributions to our understanding of the life cycle of shallow clouds and, therefore, their area fraction, cloud radiative forcing, and impact on climate.

ACKNOWLEDGMENTS. This work was supported by the U.S. Department of Energy's Atmospheric Radiation Measurement, Atmospheric System Research, and Climate Model Development and Validation programs through the Office of Science's Biological and Environmental Research program under Contract DE-AC02-05CH11231. Steven Krueger, Jordan Alpert, and two anonymous reviewers provided helpful feedback that improved the paper. Many people aided the effort to get stereo cameras installed at the ARM site, including Jennifer Comstock, Anthony Del Genio, Nicki Hickmon, Steven Klein, Steven Krueger, James Martin, James Mather, Sally McFarlane, Shaima Nasiri, Michael Ritsche, Scott Seabridge, Douglas Sisterson, Cory Stuart, David Swank, Larry Swords, and Ashley Williamson.

\section{REFERENCES}

Allmen, M. C., and P. Kegelmeyer, 1996: The computation of cloud-base height from paired whole-sky imaging cameras. J. Atmos. Oceanic Technol., 13, 97-113, https://doi.org/10.1175/1520-0426(1996)013<0097:TC OCBH $>2.0 . C O ; 2$.

Beekmans, C., J. Schneider, T. Läbe, M. Lennefer, C. Stachniss, and C. Simmer, 2016: Cloud photogrammetry with dense stereo for fisheye cameras. Atmos. Chem. Phys., 16, 14 231-14248, https://doi .org/10.5194/acp-16-14231-2016.

Bony, S., and J. Dufresne, 2005: Marine boundary layer clouds at the heart of tropical cloud feedback uncertainties in climate models. Geophys. Res. Lett., 32, L20806, https://doi.org/10.1029/2005GL023851.

Bradbury, D. L., and T. Fujita, 1968: Computation of height and velocity of mesospheric clouds from dual, whole-sky, time-lapse picture sequences. Satellite and Mesometeorology Research Project SMRP Paper 70, Dept. of Geophysical Sciences, University of Chicago, $17 \mathrm{pp}$.

Clothiaux, E. E., T. P. Ackerman, G. G. Mace, K. P. Moran, R. T. Marchand, M. A. Miller, and B. E. Martner, 2000: Objective determination of cloud heights and radar reflectivities using a combination of active remote sensors at the ARM CART sites. J. Appl. Meteor., 39, 645-665, https://doi.org/10.1175/1520 $-0450(2000) 039<0645$ :ODOCHA $>2.0 . C O ; 2$.

- - and Coauthors, 2001: The ARM Millimeter Wave Cloud Radars (MMCRs) and the Active Remote Sensing of Clouds (ARSCL) Value Added Product (VAP). DOE Tech. Memo. ARM VAP-002.1, 38 pp. + appendixes, www.arm.gov/publications/tech _reports/arm-vap-002-1.pdf.

Damiani, R., and Coauthors, 2008: The Cumulus, Photogrammetric, In Situ, and Doppler Observations experiment of 2006. Bull. Amer. Meteor. Soc., 89, 57-73, https://doi.org/10.1175/BAMS-89-1-57.

Hartmann, D. L., 2015: Global Physical Climatology. 2nd ed. Elsevier Science, $485 \mathrm{pp}$.

Hernandez-Deckers, D., and S. C. Sherwood, 2016: A numerical investigation of cumulus thermals. $J$. Atmos. Sci., 73, 4117-4136, https://doi.org/10.1175 /JAS-D-15-0385.1.

Kassander, A. R., and L. L. Sims, 1957: Cloud photogrammetry with ground-located K-17 aerial cameras. J. Meteor., 14, 43-49, https://doi.org/10.1175/0095 -9634-14.1.43.

Kassianov, E., C. N. Long, and J. Christy, 2005: Cloudbase-height estimation from paired ground-based hemispherical observations. J. Appl. Meteor., 44, 1221-1233, https://doi.org/10.1175/JAM2277.1. 
Kollias, P., E. E. Clothiaux, M. A. Miller, B. A. Albrecht, G. L. Stephens, and T. P. Ackerman, 2007: Millimeter-wavelength radars: New frontier in atmospheric cloud and precipitation research. Bull. Amer. Meteor. Soc., 88, 1608-1624, https://doi.org/10.1175/BAMS -88-10-1608.

—, N. Bharadwaj, K. Widener, I. Jo, and K. Johnson, 2014: Scanning ARM cloud radars (SACRs). Part I: Operational sampling strategies. J. Atmos. Oceanic Technol., 31, 569-582, https://doi.org/10.1175 /JTECH-D-13-00044.1.

Koppe, C., 1896: Photogrammetrie und Internationale Wolkenmessung. Braunschweig Verlag, 108 pp.

Malkus, J. S., and C. Ronne, 1954: On the structure of some cumulonimbus clouds which penetrated the high tropical troposphere. Tellus, 6, 351-366, https:// doi.org/10.1111/j.2153-3490.1954.tb01130.x.

Minor, H. A., R. M. Rauber, S. Gke, and L. Di Girolamo, 2011: Trade wind cloud evolution observed by polarization radar: Relationship to giant condensation nuclei concentrations and cloud organization. J. Atmos. Sci., 68, 1075-1096, https://doi .org/10.1175/2010JAS3675.1.

Morrison, H., and J. M. Peters, 2018: Theoretical expressions for the ascent rate of moist deep convective thermals. J. Atmos. Sci., 75, 1699-1719, https://doi .org/10.1175/JAS-D-17-0295.1.

Öktem, R., and D. M. Romps, 2015: Observing atmospheric clouds through stereo reconstruction. Proc. SPIE, 9393, 93930H, https://doi.org/10 $.1117 / 12.2083395$.

—, Prabhat, J. Lee, A. Thomas, P. Zuidema, and D. M. Romps, 2014: Stereophotogrammetry of oceanic clouds. J. Atmos. Oceanic Technol., 31, 1482-1501, https://doi.org/10.1175/JTECH-D-13-00224.1.

Orville, H. D., and A. R. Kassander, 1961: Terrestrial photogrammetry of clouds. J. Meteor., 18, 682-687, https://doi.org/10.1175/1520-0469(1961)018<0682:TP $\mathrm{OC}>2.0 . \mathrm{CO} ; 2$.

Romps, D. M., 2017: Exact expression for the lifting condensation level. J. Atmos. Sci., 74, 3891-3900, https://doi.org/10.1175/JAS-D-17-0102.1.
— - and A. B. Charn, 2015: Sticky thermals: Evidence for a dominant balance between buoyancy and drag in cloud updrafts. J. Atmos. Sci., 72, 2890-2901, https://doi.org/10.1175/JAS-D-15-0042.1.

— , and R. Öktem, 2015: Stereo photogrammetry reveals substantial drag on cloud thermals. Geophys. Res. Lett., 42, 5051-5057, https://doi.org/10 .1002/2015GL064009.

Savoy, F. M., S. Dev, Y. H. Lee, and S. Winkler, 2017: Stereoscopic cloud base reconstruction using highresolution whole sky imagers. 2017 Int. Conf. on Image Processing, Beijing, China, IEEE, 141-145, https:// doi.org/10.1109/ICIP.2017.8296259.

Seiz, G., E. P. Baltsavias, and A. Gruen, 2002: Cloud mapping from the ground: Use of photogrammetric methods. Photogramm. Eng. Remote Sensing, 68, 941-951.

Sherwood, S. C., D. Hernandez-Deckers, M. Colin, and F. Robinson, 2013: Slippery thermals and the cumulus entrainment paradox. J. Atmos. Sci., 70, 2426-2442, https://doi.org/10.1175/JAS-D-12-0220.1.

Stein, T. H. M., R. J. Hogan, P. A. Clark, C. E. Halliwell, K. E. Hanley, H. W. Lean, J. C. Nicol, and R. S. Plant, 2015: The DYMECS project: A statistical approach for the evaluation of convective storms in high-resolution NWP models. Bull. Amer. Meteor. Soc., 96, 939-951, https://doi.org/10.1175/BAMS -D-13-00279.1.

Warner, C., J. Renick, M. Balshaw, and R. Douglas, 1973: Stereo photogrammetry of cumulonimbus clouds. Quart. J. Roy. Meteor. Soc., 99, 105-115, https://doi .org/10.1002/qj.49709941910.

Wilson, J. W., and Coauthors, 1992: The role of boundary-layer convergence zones and horizontal rolls in the initiation of thunderstorms: A case study. Mon. Wea. Rev., 120, 1785-1815, https://doi. org/10.1175/1520-0493(1992)120<1785:TROBLC>2 $.0 . \mathrm{CO} ; 2$.

Zehnder, J. A., J. Hu, and A. Razdan, 2007: A stereo photogrammetric technique applied to orographic convection. Mon. Wea. Rev., 135, 2265-2277, https:// doi.org/10.1175/MWR3401.1. 